Gemeinsame Tagung der NEK-CNE und der SAMW, Freitag, 1. Juli 2011, Kongresszentrum Hotel Ador, Bern

\title{
Medizin total - Medikalisierung des Alltags als ethisches Problem
}

\begin{abstract}
Dass das Verständnis von Gesundheit und Krankheit in hohem Mass zeit- und kulturspezifisch ist, ist eine Binsenwahrheit. Dass die konkrete Einordnung bestimmter physischer oder psychischer Zustände als «gesund» oder «krank» - etwa im Bereich der Sozialversicherungen - aber sowohl für die einzelnen Betroffenen wie für die gesamte Gesellschaft weitreichende Implikationen hat, findet erst in jüngerer
\end{abstract}

\section{Programm \\ 9.15 Uhr Begrüssung und Einführung Prof. Otfried Höffe, Präsident der NEK-CNE, Tübingen Prof. Christian Kind, Präsident der Zentralen Ethikkommission der SAMW, St. Gallen \\ Praxisbeispiele \\ 9.35 Uhr Eisenmangelsyndrom - eine neue Krankheit? \\ Dr. Roch Ogier, MD-PhD, medical Director Vifor, Genève Dr. Franziska Demarmels Biasiutti, Stellvertretende Chefärztin Hämatologie, Insel-Spital Bern \\ 10.05 Uhr Burn-out-Syndrom: Diagnose oder Lifestyle? \\ PD Dr. Peter Schneider, Psychoanalytiker und Kolumnist, Zürich \\ PD Dr. Alessandra Canuto, Cheffe de service a.i., Service de psychiatrie de liaison et d'intervention de crise, Hôpitaux Universitaires de Genève \\ 10.35 Uhr Diskussion \\ 11.00 Uhr Pause}

Konzepte von Gesundheit und Krankheit 11.30 Uhr Krankheitsbilder als Spiegel ihrer Zeit PD Dr. Iris Ritzmann, Universität Zürich Gesundheit und Krankheit als normative Begriffe

Dr. Dirk Lanzerath, Deutsches Referenzzentrum für Ethik in den Biowissenschaften, Bonn

12.10 Uhr Diskussion

12.30 Uhr Mittagessen
Medikalisierung des Alltags als ethisches Problem - systematische Aspekte 13.30 Uhr Medikalisierung des Alltags: Anreize und Kosten Prof. Stefan Felder, Universität Basel

13.50 Uhr Gesellschaftliche Hintergründe der Medikalisierun Prof. Peter C. Meyer, Zürcher Hochschule für Angewandte Wissenschaften, Winterthur

14.10 Uhr Ethische Aspekte der Medikalisierung Prof. Johannes Fischer, Universität Zürich

14.30 Uhr Diskussion

15.15 Uhr Pause

15.45 Uhr Podiumsdiskussion: Ökonomisierung der Medizin - Symptom oder Ursache einer fortschreitenden Medikalisierung? Yves Rossier, Direktor, Bundesamt für Sozialversicherungen, Bern PD Dr. Iris Ritzmann, Universität Zürich Dr. Constantin Schuler, Spezialarzt für Orthopädische Chirurgie, Mörschwil Prof. Bernice Elger, Université de Genève

Dr. Dirk Lanzerath, DRZE, Bonn Moderation: Prof. Iwan Rickenbacher, Schwyz

17.00 Uhr Synthese und Ausblick Prof. Otfried Höffe, Präsident der NEK-CNE, Tübingen Prof. Christian Kind, Präsident der Zentralen Ethikkommission der SAMW, St. Gallen

17.15 Uhr Tagungsende

Simultanübersetzung Französisch-Deutsch
Zeit vermehrt Beachtung. Denn zweifellos leben wir in einer Zeit, in der, oftmals ausgehend von medizinischen Erkenntnissen, zunehmend gesellschaftlich und individuell unerwünschte Problemstellungen als Krankheiten definiert und dadurch einer medizinischen Betrachtungsweise sowie gegebenenfalls medizinischer Betreuung anheimgestellt werden. Dieser Prozess, der sowohl somatische als auch psychische Diagnosen einschliesst und - beispielsweise im Bereich der Ernährung - nicht selten auch unseren Alltag betrifft, wird als Medikalisierung bezeichnet.

Ausgehend von den konkreten Praxisbeispielen «Eisenmangelsyndrom» und «Burn-out» sowie auf der Grundlage einer historischen Einordnung soll der Prozess der Medikalisierung des Alltags anlässlich der Tagung der Nationalen Ethikkommission im Bereich Humanmedizin (NEK-CNE) und der Schweizerischen Akademie der Medizinischen Wissenschaften (SAMW) kritisch diskutiert werden. So entlastend es auf der einen Seite sein kann, das persönliche Befinden einer Diagnose zuordnen und so fassbar machen zu können, so wenig darf übersehen werden, dass die zunehmende Medikalisierung des Alltags auch einen Verlust von Autonomie und erhöhten sozialen Druck zur Folge haben kann. Dies sowohl im Hinblick auf die Möglichkeit der Inanspruchnahme medizinischer Leistungen als auch mit Blick auf die Frage nach der solidarischen Finanzierung bestimmter Therapien.

Neben den soziologischen und ethischen Aspekten der Medikalisierung widmet sich das Symposium aber auch den ökonomischen Fragen, die der Prozess der Medikalisierung des Alltags aufwirft und die auch aus ethischer Sicht von hoher Bedeutung sind. Denn die Medikalisierung eröffnet ja nicht zuletzt neue Absatzfelder, generiert aber auch nicht unwesentliche Folgekosten. Dementsprechend ist am Symposium der NEK-CNE und der SAMW auch zu diskutieren, welche wirtschaftlichen Anreize die Medikalisierung des Alltags fördern und welche volkswirtschaftlichen Kosten sie zur Folge hat. Mit Blick auf das Oberthema der Tagungsreihe wird schliesslich analysiert, wie sich die Ökonomisierung der Medizin und die Medikalisierung des Alltags wechselseitig beeinflussen.

Information/Anmeldung: www.samw.ch 